Gut, 1986, 27, 970-971

Case report

\title{
Monozygous twins concordant for duodenojejunal villous atrophy and dermatitis herpetiformis
}

\author{
S T GREEN, S NATARAJAN, J M CONNOR, AND J A H FORREST \\ From Stobhill General Hospital, Glasgow, and the Duncan Guthrie Institute of Medical Genetics, Yorkhill, \\ Glasgow
}

SUMMARY A pair of monozygous twins concordant for both duodenojejunal villous atrophy and dermatitis herpetiformis are described, the first example to be reported in the literature. The observation provides strong support for the concept of an underlying genetic component being involved in the pathogenesis of these two conditions.

Duodenojejunal villous atrophy, morphologically identical to that seen in coeliac disease, ${ }^{\text {is encount- }}$ ered in association with two-thirds of cases of dermatitis herpetiformis. ${ }^{1}$ Although the duodenojejunal villous atrophy of dermatitis herpetiformis does respond to a gluten free diet, ${ }^{2}$ however, clinically detectable malabsorption is rare. ${ }^{i}$ Although the concordance rate among identical twins for coeliac disease is virtually $100 \%,{ }^{3}$ only a single pair of male monozygotic twins both suffering from clinically diagnosed dermatitis herpetiformis (with no evidence of duodenojejunal villous atrophy) have been reported, and neither brother was subjected to skin or jejunal biopsy. ${ }^{4} \mathrm{We}$ therefore wish to report a pair of female monozygous twins with histologically proven dermatitis herpetiformis and duodenojejunal villous atrophy.

\section{Case reports}

CASE ONE

A 53 year old Scottish woman presented in 1964 with a 10 year history of an intermittent blistering itchy rash. Dapsone was started with good symptomatic effect. A flare up in 1980 prompted a skin biopsy and granular $\operatorname{IgA}$ deposition in the dermal papillae confirmed a diagnosis of dermatitis herpetiformis. In January 1984, she developed diarrhoea and weight loss. Serum and red cell folate levels were subnormal and a small bowel biopsy revealed subtotal villous atrophy, crypt hyperplasia, and an

Address for correspondence: Dr Stephen T Green, Department of Materia Medica, Stobhill General Hospital, Glasgow G21.

Received for publication 21 November 1985. increased intraepithelial lymphocyte count. Her gastrointestinal symptoms settled on a gluten free diet but she refused a repeat small bowel biopsy.

\section{CASE TWO}

The twin sister of case one, who was strikingly similar in physical appearance to her, presented in 1970 with a 14 year history of a recurring pruritic vesicular rash which responded well to dapsone. In May 1985, a skin biopsy revealed granular IgA deposition, concentrated at the dermal papillary tips, and a small bowel biopsy showed severe partial villous atrophy, crypt hyperplasia and an increased intraepithelial lymphocyte count. Serum folate levels were normal. The patient refused a gluten free diet but has remained free of cutaneous symptoms on dapsone. At no point in her illness has she been troubled by gastrointestinal symptoms.

Monozygosity of the sisters was supported by identical blood groups for ABO, MNS, Pi, Rh, Kell, $\mathrm{Le}^{\mathrm{a}}$, Chilano, $\mathrm{Fy}^{\mathrm{a}}, \mathrm{Fy}^{\mathrm{b}}, \mathrm{Jk}^{\mathrm{a}}, \mathrm{Jk}^{\mathrm{b}}, \mathrm{Co}^{\mathrm{a}}$ and $\mathrm{Co}^{\mathrm{b}}$.

\section{Discussion}

This is the first reported pair of monozygous twins in which both individuals have been shown to have both dermatitis herpetiformis and duodenojejunal villous atrophy. The multifactorial aetiology of duodenojejunal villous atrophy without dermatitis herpetiformis - that is, coeliac disease, is generally accepted. As dermatitis herpetiformis is rare, however (with a prevalence of 1:80 $000^{4}$ ), there have been relatively few well documented family studies, ${ }^{45}$ but these studies have appeared to show an increased risk among family members for 
dermatitis herpetiformis. The twins reported here, with their proven monozygosity, provide evidence for a genetic component in the pathogenesis of dermatitis herpetiformis, although their different disease patterns despite their identical genes indicate the importance of environmental factors in the disease.

The relative contributions of genetic factors - for example, HLA status, and environmental agents for example, dietary gluten, in coeliac disease have been confirmed and investigated using detailed family studies. ${ }^{35}$ The situation with respect to the pathogenesis of dermatitis herpetiformis is more complex - the syndrome differs from coeliac disease in that it possesses dermatological features (which are responsive to a gluten free $\operatorname{diet}^{6}$ ) while the bowel lesions cause clinically significant malabsorption much less frequently than is the case with coeliac disease $^{1}$ : interestingly the duodenojejunal villous atrophy of dermatitis herpetiformis does not respond to the drugs used to treat the skin lesions. ${ }^{1}$ HLA associations (similar to those encountered in coeliac disease) and a variety of immunological abnormalities have been described in dermatitis herpetiformis, ${ }^{7-10}$ but despite considerable speculation as to the nature of the underlying immunopathogenesis of dermatitis herpetiformis, several central aspects of the puzzle remain to be resolved. ${ }^{70} \mathrm{~A}$ detailed discussion of the various hypotheses is beyond the scope of a case report, and the reader is referred to more detailed reviews. ${ }^{710}$

Dermatitis herpetiformis is responsible for considerable morbidity and there is a possible relationship to lymphoma and carcinoma, ${ }^{11}$ thus greater understanding of the pathogenesis of this condition would be highly desirable.

We wish to thank Drs R S Chapman and D C Dick for their advice and support, and the technical staff of the Medical Genetics, Histology, and Immunodermatology laboratories of the Duncan Guthrie Institute, Stobhill General Hospital and the Western Infirmary of Glasgow for their assistance.

\section{References}

1 Brow JR, Parker F, Rubin CE. The small intestinal mucosa in dermatitis herpetiformis I. Severity a:d distribution of the small intestinal lesion and associated malabsorption. Gastroenterology 1971; 60: 355-61.

2 Weinstein WM, Brow JR, Parker F, Rubin CE. The small intestinal mucosa in dermatitis herpetiformis II. Relationship of the small intestinal lesion to gluten. Gastroenterology 1971; 60: 362-9.

3 Greenberg DA, Lange KL. A maximum likelihood test of the two locus model for coeliac disease. Am J Med Genet 1982; 12: 75-82.

4 Marks J, May SB, Roberts DF. Dermatitis herpetiformis occurring in monozygous twins. $\mathrm{Br} \mathrm{J}$ Dermatol 1971; 84: 417-9.

5 Alexander JO'D. Dermatitis herpetiformis. London, Philadelphia \& Toronto: WB Saunders, 1975.

6 Fry L, Seah PP, Riches DJ, Hoffbrand AV. Clearance of skin lesions in dermatitis herpetiformis after gluten withdrawal. Lancet 1973; 1: 288-91.

7 Katz SI, Hall RP, Lawley TJ, Strober W. Dermatitis herpetiformis: the skin and the gut. Ann Intern Med 1980; 93: 857-74.

8 Chorlzelski TP, Beutner EH, Sulej J, et al. IgA antiendomysium antibody: a new immunological marker of dermatitis herpetiformis and coeliac disease. $\mathrm{Br} J$ Dermatol 1984; 111: 395-402.

9 Maury CJP, Teppo A-M. Demonstration of tissue 90 $\mathrm{kD}$ glycoprotein as antigen in circulating IgG immune complexes in dermatitis herpetiformis and coeliac disease. Lancet 1984; 2: 892-4.

10 Huff JC. The immunopathogenesis of dermatitis herpetiformis. J Invest Dermatol 1985; 84: 237-8.

11 Leonard JN, Tucker WFG, Fry JS, et al. Increased incidence of malignancy in dermatitis herpetiformis. Br Med J 1983; 286: 16-8. 